\title{
The Effects of Ambient Stratification on the Crow Instability and Subsequent Vortex Reconnection
}

\author{
J. F. Garten, J. Werne, D. C. Fritts, S. Arendt \\ Colorado Research Associates, 3380 Mitchell Lane, Boulder, CO 80301, United States. \\ James.Garten@colorado-research.com
}

We discuss the results of direct numerical simulations of three-dimensional (3D) anti-parallel vortex pairs evolving in ambient stratification for a range of Reynolds $(R e)$ and Froude $(F r)$ numbers. These results have relevance to both the study of aircraft trailing vortices, which can pose considerable hazard to following aircraft (see, e.g., Olsen et al. 1971), and the general topic of vortex reconnection, an important process in fully-developed turbulence and other complex flows (see, e.g., Rogers \& Moin 1988, Melander \& Hussain 1988).

\section{Introduction}

Our purpose here is to investigate how stratification affects the evolution of anti-parallel vortices, from their initial evolution as it is controlled by the Crow instability, through their reconnection into vortex rings, and subsequent aspects of the evolution. As different processes are dominant during different phases of the evolution, we separately discuss Crow instability $(0<t \lesssim 3.5)$ and vortex reconnection $(3.5 \lesssim t \gtrsim 6$ to 8 , depending on $R e$ and Fr) phases.

Using a linear perturbation analysis of anti-parallel vortex tubes (with constant cross-section) in an unstratified and inviscid fluid, Crow (1970) found that the most unstable mode of instability depends on the ratio of the radius of the vortex cores $r_{C}$ to their separation distance $b_{0}$. For $r_{C} / b_{0}=0.098\left(d / b_{0}=0.063\right.$ in Crow's notation $)$, the fastest growing mode is symmetric, has a wavelength of $8.6 b_{0}$, amplifies with an $e$-folding time of $T=1.21 b_{0} / W_{0}\left(W_{0}\right.$ is the initial induced vertical velocity), and develops on each tube in a plane tilted $48^{\circ}$ from the horizontal. We use these values in our initial conditions (

Most work concerning the effects of ambient stratification on vortex pairs has unfortunately been limited to the study of two-dimensional (2D) vortex pairs, which cannot undergo the 3D Crow instability. Recent numerical simulations by Spalart (1996) and Garten et al. (1998) have shown that weak ambient stratification $(F r \geq 1)$ causes a decrease in separation distance and subsequent acceleration of laminar 2D vortex pairs, in general agreement with the theoretical predictions of Crow (1974) and Scorer \& Davenport (1970), and the experimental results of Tomassian (1979). Nevertheless, this 2D result may not be relevant to 3D flows because the Crow instability may dominate the evolution on a time scale that precludes the $(2 \mathrm{D})$ acceleration from occurring. In fact, we know of no instances in which acceleration of a 3D vortex pair in stratification has been observed in experiments (e.g., Sarpkaya 1983) or numerical simulations (e.g., Robins \& Delisi 1997). For the 3D solutions reported here, we also find no acceleration of 3D vortex pairs; however, we do find that the decrease in separation distance persists from the 2D case, and that this influences the growth of the Crow instability (and the subsequent evolution).

Once the instability reaches a large enough amplitude for portions of the vortex tubes to come into close contact, vortex reconnection commences, and, in general, the vortex tubes are rearranged 
into a series of vortex rings. In aviation safety terms (see, e.g., Spalart 1998), the underlying questions concern the time evolution of the circulation of these vortex rings, their trajectories, and how quickly the energy of the flow dissipates. Aside from its importance in the evolution of aircraft trailing vortices, vortex reconnection has also been conjectured to play important roles in the mixing and production of turbulence, helicity, and aerodynamic noise (Hussain 1986). Much work has been done on the topic of vortex reconnection in the context of anti-parallel vortex tubes undergoing reconnection in an unstratified fluid, including Pumir \& Kerr (1987), Melander \& Hussain (1989), Buntine \& Pullin (1989), Kerr \& Hussain (1989), Saffman (1990), Kida et al. (1991a), and Shelley et al. (1993). The only work of which we are aware that explicitly discusses the reconnection of anti-parallel vortex tubes in a different (e.g., stratified) background are the compressible calculations of Virk et al. (1995), who found that compressible effects initially enhance reconnection, but later slow it down. With our Boussinesq (i.e., incompressible) calculations, we find that ambient stratification results in an acceleration of the reconnection process and a loss in the total circulation of the vortex tubes due to cancellation with baroclinic vorticity.

\section{$2 \quad$ Numerical Method}

We use a numerical model based on the Boussinesq approximation to the 3D Navier-Stokes equations; a non-dimensional and efficient form of these equations is:

$$
\begin{gathered}
\boldsymbol{\nabla} \cdot \mathbf{v}=0 \\
\frac{\partial}{\partial t} \nabla^{2} w=\hat{\mathbf{z}} \cdot \boldsymbol{\nabla} \times \boldsymbol{\nabla} \times(\boldsymbol{\omega} \times \mathbf{v})+\frac{1}{F r^{2}} \nabla_{\perp}^{2} T+\frac{2 \pi}{R e} \nabla^{4} w \\
\frac{\partial \omega_{z}}{\partial t}=-\mathbf{v} \cdot \nabla \omega_{z}+\boldsymbol{\omega} \cdot \boldsymbol{\nabla} w+\frac{2 \pi}{R e} \nabla^{2} \omega_{z} \\
\frac{\partial}{\partial t} \mathbf{U}_{\perp}=-\frac{\partial}{\partial_{z}}\left\langle w \mathbf{v}_{\perp}\right\rangle+\frac{2 \pi}{R e} \frac{\partial^{2}}{\partial z^{2}} \mathbf{U}_{\perp} \\
\frac{\partial T}{\partial t}=-(\mathbf{v} \cdot \nabla)(\bar{T}+T)+\frac{2 \pi}{R e \operatorname{Pr}} \nabla^{2} T
\end{gathered}
$$

where $\mathbf{v}=(u, v, w)$ is the velocity; $\boldsymbol{\omega}=\boldsymbol{\nabla} \times \mathbf{v}=\left(\omega_{x}, \omega_{y}, \omega_{z}\right)$ is the vorticity; $\perp$ denotes the projection onto the horizontal plane, \langle\rangle denotes the horizontal average, and $\mathbf{U}_{\perp}$ is the mean horizontal velocity. Equations (1)-(5) have been non-dimensionalized by the initial vortex separation distance $b_{0}$, the induced vertical velocity $W_{0}$, and the background temperature gradient $\beta$, where $N^{2}=g \alpha \beta, g$ is the acceleration due to gravity, and $\alpha$ is the thermal expansion coefficient. Useful combinations of these variables include the Froude number, $\mathrm{Fr}=W_{0} / N b_{0}$, the Reynolds number, $R e=2 \pi W_{0} b_{0} / \nu=\Gamma_{0} / \nu$, and the Prandtl number, $\operatorname{Pr}=\nu / \kappa$. We solve this system of equations with a pseudo-spectral, stream-function/vorticity, Galerkin method (see, e.g., Canuto et al. 1988), using a hybrid implicit/explicit 3rd-order Runga-Kutta time-stepping scheme (Spalart et al. 1991).

Our initial conditions consist of two anti-parallel vortex tubes with Gaussian cross section (e.g., $\omega \sim e^{-r^{2} / 2 \sigma^{2}} ; \sigma=0.72 r_{C}$ yields a vortex with the same peak vorticity and total circulation as a vortex with constant cross section), whose centers are initially positioned in a perturbed, though symmetric, configuration to excite the Crow instability. We take advantage of the symmetry by only computing half of the total solution, and Table 1 provides a summary of information for the simulations that we discuss here. 


\begin{tabular}{|cccc|ccc|ccc|}
\hline \hline \multicolumn{3}{|c|}{ Flow Parameters } & \multicolumn{3}{c|}{ Domain Lengths } & \multicolumn{3}{|c|}{ Spectral Modes } \\
$F r$ & $\sigma / b_{0}$ & $R e$ & $\operatorname{Pr}$ & $L_{x}$ & $L_{y} / 2$ & $L_{z}$ & $N_{x}$ & $N_{y}$ & $N_{z}$ \\
\hline$\infty$ & .0707 & 628 & 1 & 8.6 & 4 & 8 & 128 & 128 & 256 \\
$\infty$ & .0707 & 942 & 1 & 8.6 & 4 & 8 & 256 & 192 & 384 \\
$\infty$ & .0707 & 942 & 1 & 8.6 & 8 & 8 & 256 & 384 & 384 \\
$\infty$ & .0707 & 1260 & 1 & 8.6 & 4 & 8 & 256 & 256 & 512 \\
8 & .0707 & 942 & 1 & 8.6 & 8 & 10.67 & 256 & 384 & 512 \\
4 & .0707 & 942 & 1 & 8.6 & 8 & 10.67 & 256 & 384 & 512 \\
2 & .0707 & 942 & 1 & 8.6 & 8 & 10.67 & 256 & 384 & 512 \\
1 & .0707 & 942 & 1 & 8.6 & 8 & 10.67 & 256 & 384 & 512 \\
$1 / 2$ & .0707 & 942 & 1 & 8.6 & 8 & 10.67 & 256 & 384 & 512 \\
\hline \hline
\end{tabular}

\section{Crow Instability in Ambient Stratification}

As we are interested in the effects of environmental factors, like ambient stratification, on the growth of the Crow instability, we first consider how it evolves in the absence of external effects. Fig. 1 shows the time evolution of the instability amplitude for three unstratified cases. These results clearly show that the qualitative agreement with Crow's prediction improves with increasing $R e$. Nevertheless, the comparison is imperfect. First, the numerical solutions do not duplicate exactly the vortex tube topology predicted by linear theory, as vortex tube displacements are not perfectly sinusoidal (see, e.g., Moore 1972). Second, viscous diffusion plays an important role in the evolution of the instability, especially at small $R e$. Since Crow's model is inviscid, we cannot expect it to describe the Re dependence; however, and as discussed in more detail in a forthcoming paper, the slower growth of the instability for smaller $R e$ can be predicted from the significant increase in vortex core size via viscous diffusion during the growth of the instability.

Fig. 2 shows the time evolution of the instability in greater detail than presented in Fig. 1. The mean inclination angle $(\theta)$ of the vortex tube displacements is plotted versus time in Fig. 2a, and it is compared to the predicted evolution of this angle when Crow's model is extended to include diffusion of the vortex core. In each case, $\theta$ increases slowly with time in qualitative agreement with the extended model's predictions. Fig. $2 \mathrm{~b}$ shows displacements of the vortex core projected onto the plane of mean inclination $\left(a_{C}\right)$, and Fig. 2c shows displacements perpendicular to this plane $\left(d_{C}\right)$. The two locations along the vortex tubes we have chosen to present in Fig. 2b and Fig. 2c correspond to the positions of furthest separation (star symbol) and closest approach (diamond symbol) of the vortex tubes. In Fig. $2 \mathrm{~b}, a_{C}$ is compared to the predictions of Crow's model with fixed vortex core size (dashed line), and our modified Crow's model which includes diffusion of the vortex core (dotted line). The overall agreement with the modified theory is best for the $R e=628$ case and slightly worsens as $R e$ increases. We believe the increased discrepancy at larger $R e$ indicates the reduction in importance of diffusive core growth relative to nonlinear effects. In Fig. 2c, the time evolution of $d_{C}$ (multiplied by 10) shows the increasing distortion of the tube displacements from sinusoidal shapes for larger $R e$, and this may further impact our ability to predict the instability growth with our modified theory. Most of the distortion results from a variation in the vertical propagation of the vortex tubes caused by the variation in separation distance along the tubes (i.e., the tubes are elongated in the vertical direction); therefore, the 


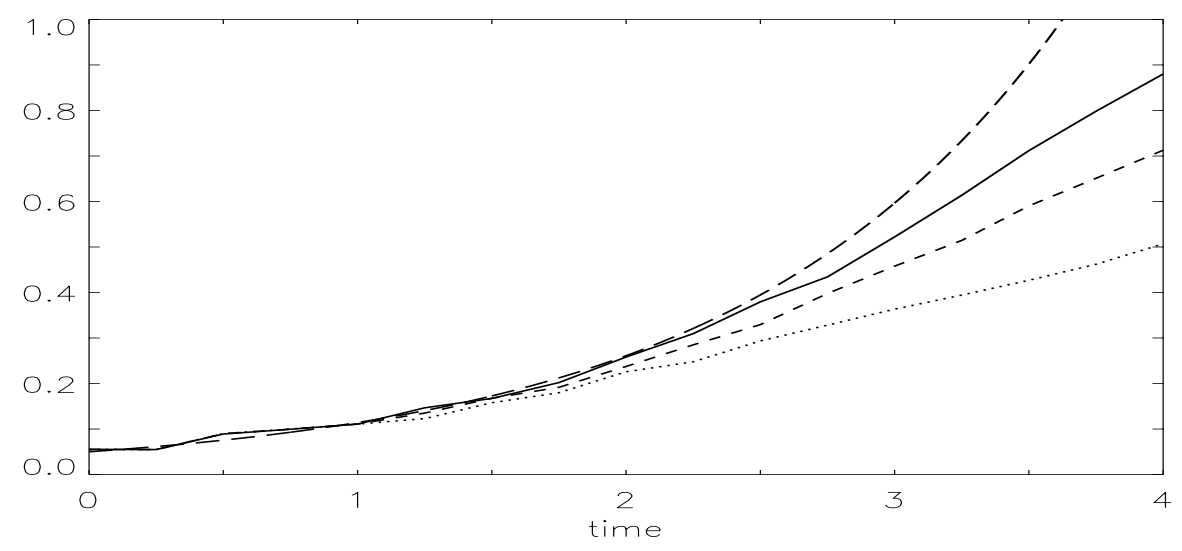

Figure 1: The time evolutions of the instability amplitude for the unstratified cases with $R e=628$ (dotted), $R e=943$ (short-dashed), and $R e=1257$ (solid), compared to the theoretical prediction of Crow's model (long-dashed).

displacement amplitude is henceforth calculated by projecting the horizontal displacements only onto the plane of mean inclination.

We now consider how ambient stratification affects the development of the Crow instability. A simple time-scale analysis suggests that this part of the evolution should not be significantly affected unless $F r \sim O(1)$ or smaller: in the non-dimensional time units, the buoyancy period is $2 \pi / N=2 \pi F r$, while our initial amplitude leads to "saturation" of the instability (i.e., the instability reaches a large enough amplitude for significant vortex reconnection to have commenced) by about $t \simeq 3.5$ (see next section). The time evolutions of the instability amplitude for four of our stratified cases (all with $R e=943$ ) are shown in Fig. 3, and they demonstrate this simple expectation. The growth of the instability for $F r \geq 2$ is nearly identical to the unstratified $(F r \rightarrow \infty)$ case, while it grows noticeably faster for $F r=1$, and noticeably slower for $F r=1 / 2$.

In measuring the instability amplitude in these stratified cases, it is important to account for the time dependence of the average vortex tube separation distance. Therefore, the displacements of the vortex cores must be measured with respect to their time evolving average positions. The theoretical predictions of Scorer \& Davenport (1970) and Crow (1974), and the results of recent large- $R e$ numerical simulations of 2D vortex pairs in ambient stratification by Spalart (1996) and Garten et al. (1998) provide explanations for changes in the separation distance with time. Horizontal density gradients result from the downward transport of relatively lighter fluid by the vortices; these gradients constitute a baroclinic source that generates vorticity behind and to the sides of the original vortex tubes. For $F r \geq 1$, the regions of this secondary vorticity advect 

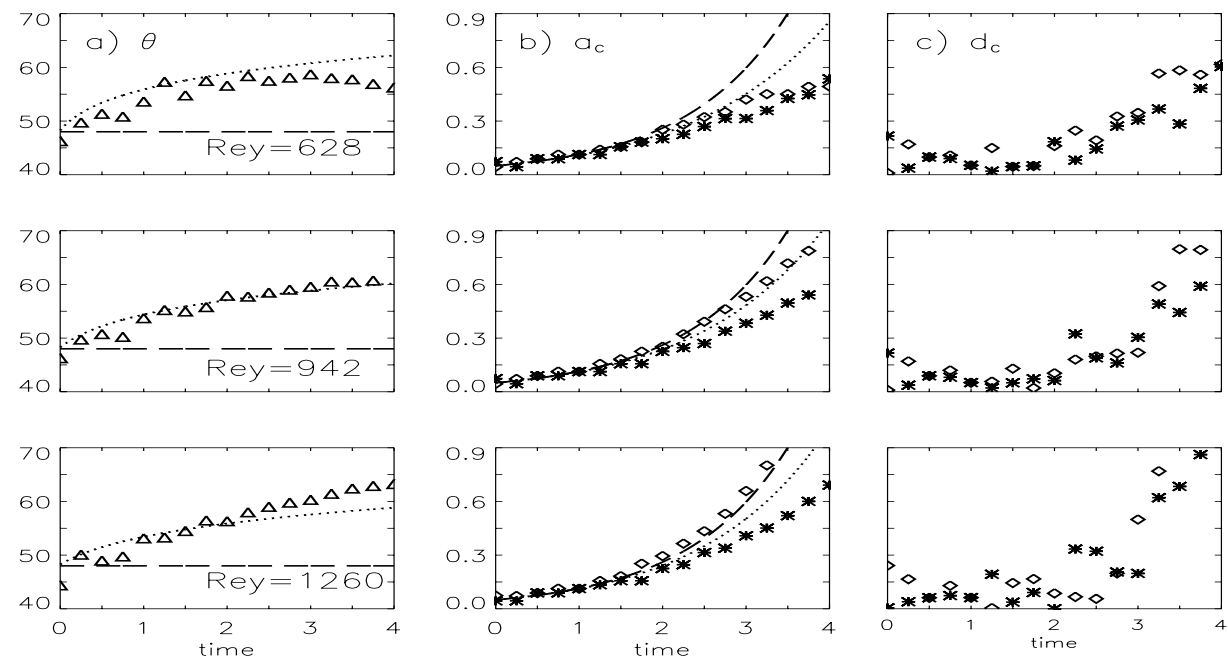

Figure 2: The time evolutions of a) the mean inclination angle of the vortex tube displacements, b) the amplitude of the displacements projected onto the plane of mean inclination, and c) the amplitude of the displacements perpendicular to this plane (multiplied by 10), for the unstratified cases with $R e=628$ (top), $R e=943$ (middle), and $R e=1257$ (bottom). The vortex core displacements have been measured at the positions of furthest separation (star symbol) and closest approach (diamond symbol) of the vortex tubes, and comparisons to the predictions of the original (dashed) and modified (dotted) Crow models are shown.
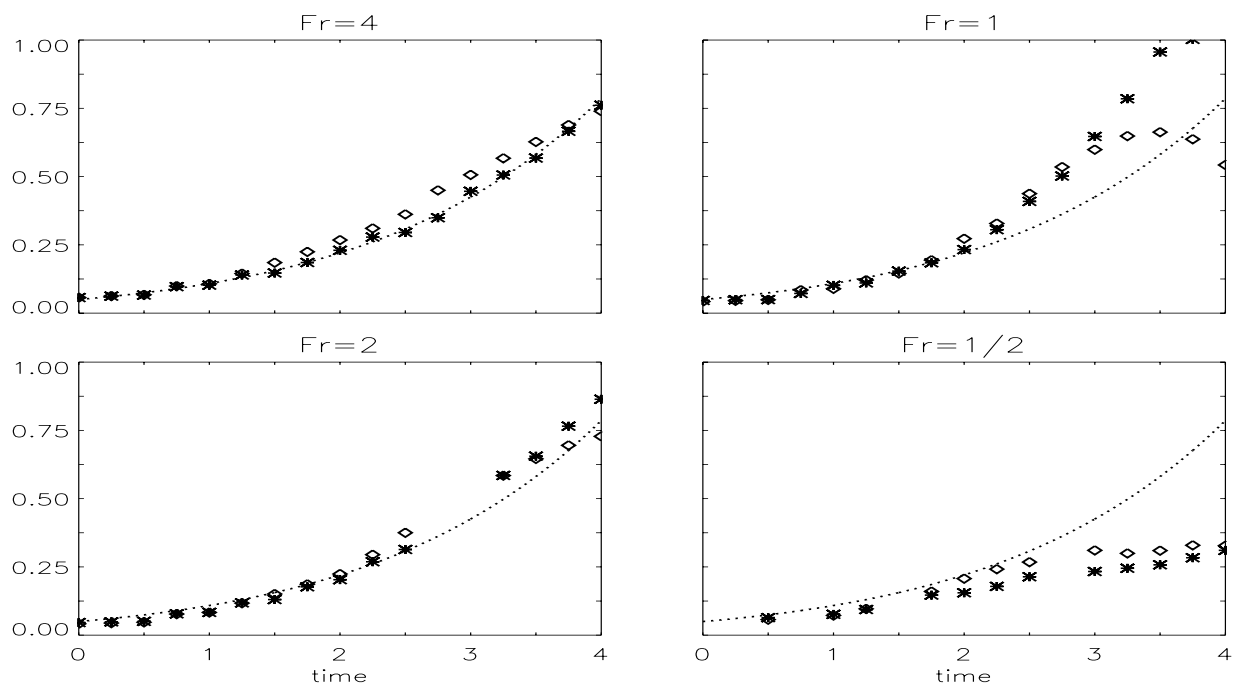

Figure 3: The time evolution of the instability amplitude as given by the horizontal displacements of the vortex cores for the stratified cases with $R e=943$ and $F r=4$ (top left), $F r=2$ (bottom left), $F r=1$ (top right), and $F r=1 / 2$ (bottom right). The displacements are measured in the spanwise planes of furthest separation (star symbol) and closest approach (diamond symbol) of the vortex tubes, and comparisons to the predictions of the modified Crow model (dotted lines) are shown. 

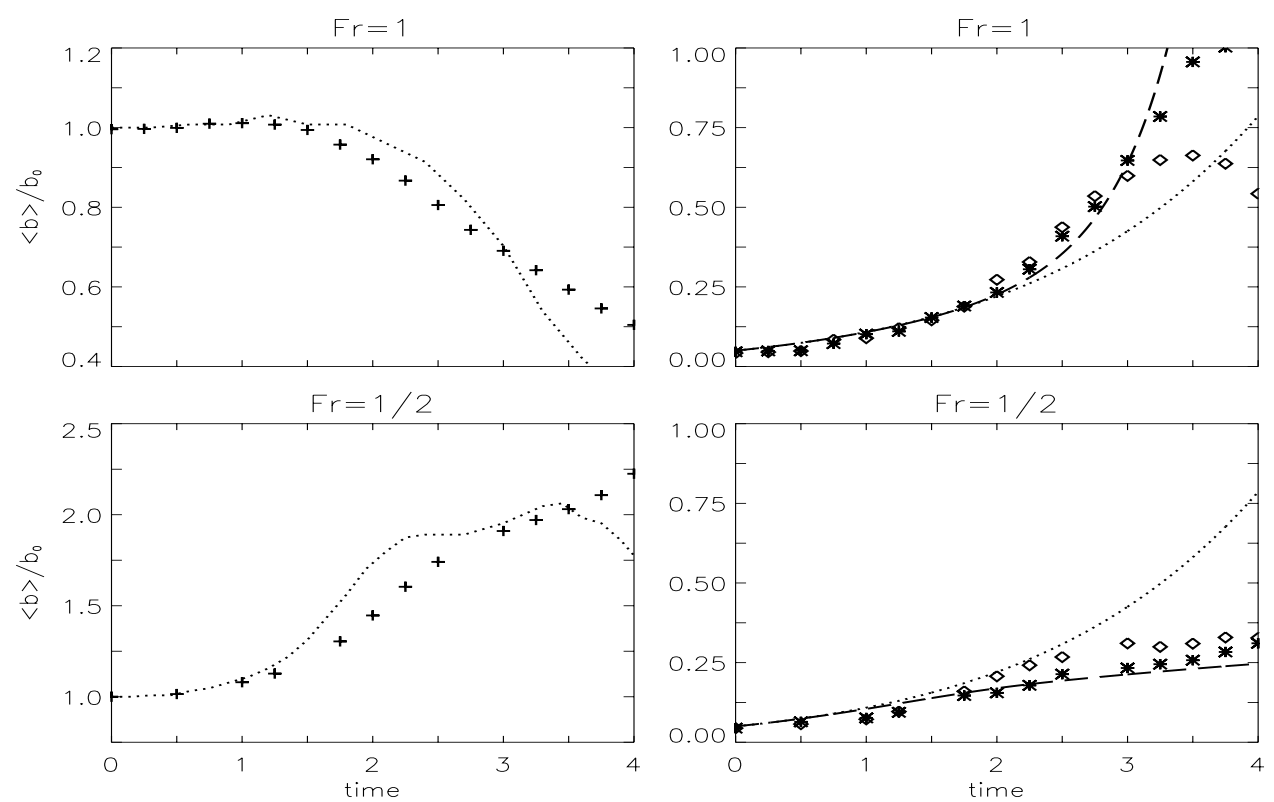

Figure 4: The time evolutions of the average vortex separation distance (left) and the instability amplitude as given by the horizontal perturbations of the vortex cores (right) for the stratified cases with $R e=943$ and $F r=1$ (top) and $F r=1 / 2$ (bottom). On the left, the data is compared to the results of simulations of $2 \mathrm{D}$ vortex pairs at the same $\operatorname{Fr}$ (dotted lines), while on the right, comparisons to the predictions of the modified Crow model (dotted lines) and the modified Crow model including a changing $\langle b\rangle$ (dashed lines) are shown.

the primary vortices towards one another, while for $F r \leq 1 / 2$ they (have a qualitatively different distribution and) advect the primary vortices away from one another (see Fig. 4). For our cases with $F r \geq 4$, the average separation distance of the vortex tubes changes by less than $5 \%$ through $t=4$, while for our cases with $F r \leq 2$, more rapid changes occur.

The change in average separation distance of the vortex tubes $(\langle b\rangle)$ suggests a somewhat naive explanation for the time-evolving growth of the Crow instability. As $\langle b\rangle$ evolves, the time scaling of the evolution (recall that time is non-dimensionalized by $b_{0} / W_{0}$, and that $W_{0} \sim 1 / b_{0}$ ) proceeds as $\delta t \sim 1 /\langle b\rangle^{2}$. Including this "acceleration" factor (a deceleration for the $F r=1 / 2$ case in which $\langle b\rangle$ increases) in our modified Crow model, we can attempt to predict the growth of the instability for $F r \sim O(1)$; see Fig. 4. The qualitative agreement is good, though it degrades for $F r=1$ after $t \simeq 3$ when the proximity of the vortex tubes begins to restrict the size of the horizontal displacement.

\section{Vortex Reconnection in Ambient Stratification}

As we are interested in the effects of environmental factors, like ambient stratification, on the vortex reconnection phase of the evolution, we again consider first what occurs in the absence of external effects. We introduce the following notation to characterize the speed of reconnection: $\Gamma_{f}(t)$ is 

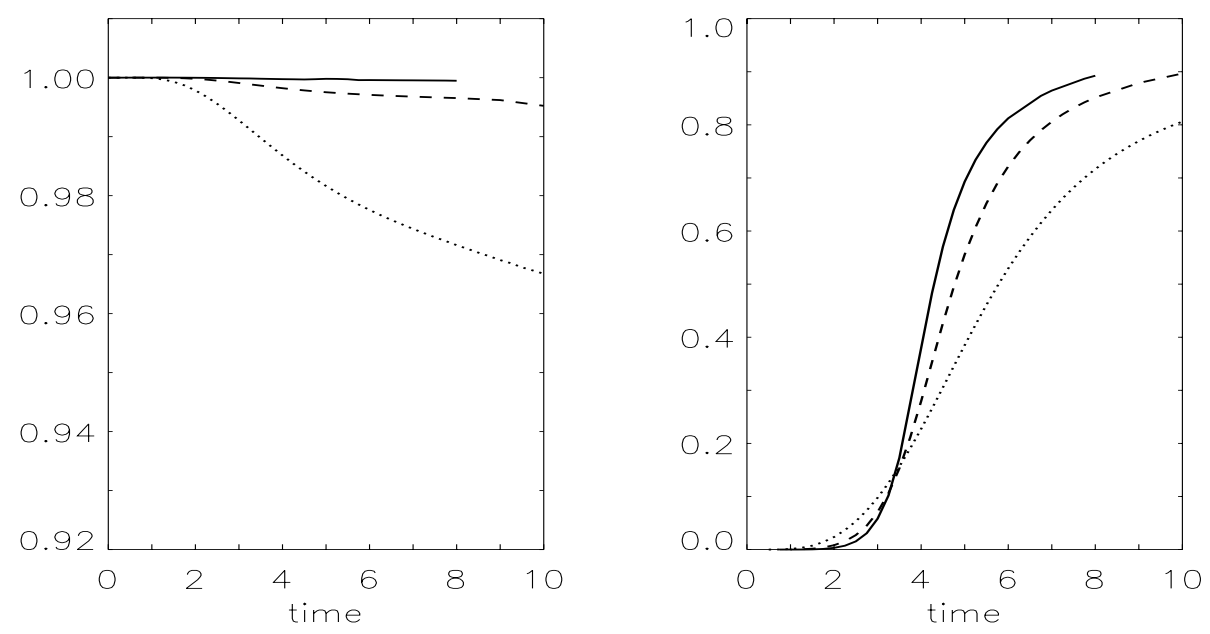

Figure 5: The time evolutions of the undisturbed circulation of each vortex tube $\left(\Gamma_{f}(t) / \Gamma_{0}\right.$, left $)$ and the fraction of reconnected circulation $\left(\Gamma_{r}(t) / \Gamma_{f}(t)\right.$, right $)$ for the unstratified cases with $R e=628$ (dotted), $R e=943$ (short-dashed), and $R e=1257$ (solid).

the total circulation of the positive vortex tube at the location of furthest separation of the vortex tubes (the spanwise plane that cuts across the vortex tubes at this location is henceforth referred to as plane $f$ ). Due to the symmetries of the flow, the total circulation of the other vortex tube at this location is $-\Gamma_{f}(t)$, and $\Gamma_{f}(t)$ can only decrease (in the unstratified case) via cross-diffusion at the centerplane between the vortices. Calling $\Gamma_{c}(t)$ the circulation at the location of closest approach of the vortex tubes (the spanwise plane $c$ cuts across the vortex tubes at this location), the flow's symmetries also dictate that $\Gamma_{f}(t)-\Gamma_{c}(t)=\Gamma_{r}(t)$, where $\Gamma_{r}(t)$ is the total circulation of reconnected vorticity. The regions of vorticity that are formed from the realignment of the non-annihilated parts of vortex lines whose parts in and near the spanwise plane $c$ are annihilated via cross-diffusion, and in a sense connect one vortex tube to the other (forming a vortex ring) have been called bridges by Melander \& Hussain (1989). Plots of $\Gamma_{f}(t)$ normalized by the initial circulation and $\Gamma_{r}(t) / \Gamma_{f}(t)$ are shown in Fig. 5.

As given by the time evolution of $\Gamma_{f}$, there is a small but noticeable total loss in circulation (i.e., some cross-diffusion occurs everywhere along the tubes). This is a consequence of the increase in size of the vortex cores (through viscous diffusion): some anti-parallel vorticity cancels in plane $f$ even though the growth of the Crow instability results in migration of the core centers away from one another (and the centerplane). We note that this loss was avoided by Melander \& Hussain (1989), who used compact-Gaussian vorticity profiles in their simulations. For our simulations, a larger Re results in slower growth for the vortex core size, and in more rapid growth for the instability amplitude; both of these effects reduce the vorticity gradient at the centerplane, reducing 
the cross-diffusion. For increasing $R e$, the percentage of the original circulation that is lost by $t=8$ is $2.8 \%, 0.3 \%$, and $0.1 \%$, respectively.

The time evolution of $\Gamma_{r} / \Gamma_{f}$ (see Fig. 5) indicates that the process of vortex reconnection occurs more rapidly and leads to a larger fraction of reconnected circulation for larger $R e$ (for our range of $R e$ ). This appears to result from faster instability growth and the associated enhanced vorticity gradients at the centerplane. Even though $R e$ is larger, where cross-diffusion (resulting in reconnection) might be expected to occur more slowly, it occurs more rapidly because the gradients become much larger and are also spread over a larger vertical extent because of the characteristic "head-tail" deformation (see, e.g., Kida et al. 1991b) in and near plane $c$.

When stratification is present, $\Gamma_{f}$ decreases precipitously (see Fig. 6). The sharp decrease is important to understand, as it may support a "predictable decay" (PD) view of the evolution (see Spalart, 1998, for a full explanation of the PD and "stochastic collapse" (SC) view points in the context of airplane trailing vortices), in which it is assumed that the circulation gradually decays in a manner easily predicted from the environmental conditions. There are two possible mechanisms that could contribute to this decrease: 1) enhanced cross-diffusion in plane $f$ resulting from the advection of the primary vortices towards one another by the baroclinic vorticity (as discussed in $\S 3$ ), or 2) cancellation with the countersigned baroclinic vorticity itself. As discussed more fully in a forthcoming paper, we have determined that it is cancellation with the baroclinic vorticity that leads to the loss in $\Gamma_{f}$; smaller $F r$ result in more rapid formation of baroclinic vorticity, and the subsequent rapid decrease in the circulation of the primary vortex. Furthermore, we have determined from simulations of $2 \mathrm{D}$ vortex pairs in ambient stratification for a range of $R e$ that the impact of this cancellation substantially decreases with increasing $R e$. Therefore, caution should be exercised when contemplating the results summarized in Fig. 6; specifically, one cannot conclude that our results directly support a PD view.

Even though the circulation of the vortex tubes decreases more rapidly for smaller $\mathrm{Fr}$, the reconnection proceeds more rapidly (i.e., the fraction $\Gamma_{r} / \Gamma_{f}$ increases more rapidly). This results from the advection of the primary vortices towards one another by the baroclinic vorticity (in effect, the baroclinic vorticity provides an external straining field that drives the vortices towards the centerplane); in and near plane $c$, the vorticity gradients steepen more quickly, and the rate of cross-diffusion is enhanced. For $F r=2$ and $F r=1$, the continuous advection by the baroclinic vorticity results in $\Gamma_{c} \rightarrow 0$ (and hence $\Gamma_{r} / \Gamma_{f} \rightarrow 1$ ), so that in this sense the reconnection could be viewed as "complete" (within a dynamically interesting time frame). However, for the larger $\mathrm{Fr}$ cases (including $\mathrm{Fr} \rightarrow \infty$ ), $\Gamma_{c}$ retains a nonzero value when a flow induced by the vortex bridges retards the annihilation of the vortex tubes (Melander \& Hussain 1989). The slender portions of the vortex tubes that survive outside of the bridges have been called threads by Melander \& Hussain (1989), who speculated that under the right conditions, these threads could undergo a secondary reconnection process.

\section{Summary}

We have found that ambient stratification can substantially affect the evolution of anti-parallel vortex pairs undergoing the Crow instability and subsequent vortex reconnection. Our unstratified 

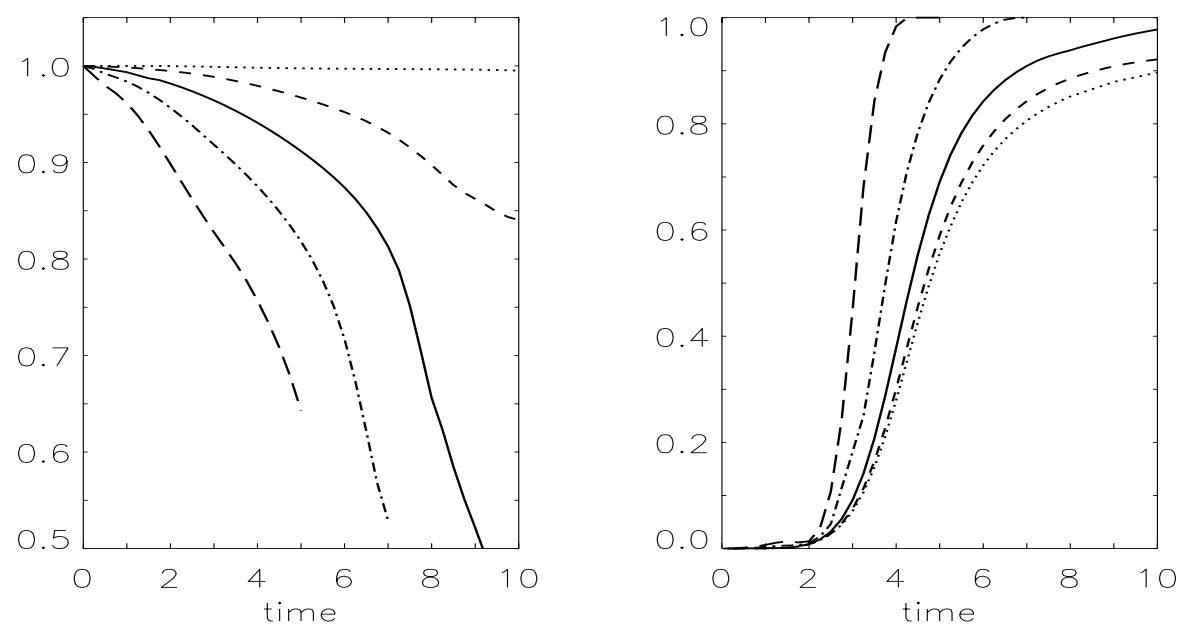

Figure 6: The time evolutions of the undisturbed circulation of each vortex tube $\left(\Gamma_{f}(t) / \Gamma_{0}\right.$, left) and the fraction of reconnected circulation $\left(\Gamma_{r}(t) / \Gamma_{f}(t)\right.$, right $)$ for the $R e=943$ cases with $\mathrm{Fr} \rightarrow \infty$ (dotted), $F r=8$ (short-dashed), $F r=4$ (solid), $F r=2$ (dash-dotted), and $F r=1$ (long-dashed).

simulations show that the growth rate of the Crow instability has some Re dependence in the range of $R e$ we are able to access, and we present the concept for a simple extension to Crow's model to explain and predict this dependence. We find that relatively strong ambient stratification $(F r \leq 2)$ further alters the growth of the Crow instability when the baroclinic vorticity either advects the primary vortices towards one another $(F r \geq 1)$, leading to a faster growth, or away from one another $(F r \leq 1 / 2)$, retarding the growth and preventing significant vortex reconnection from ever commencing. For the latter regime, the growth of the 3D instability is sufficiently slowed that a 2D description will become increasingly sufficient for smaller $\mathrm{Fr}$; see Jaderberg (1980), Garten (1997), and Garten et al. (1998) for studies of 2D vortex pairs in strong ambient stratification.

Our stratified simulations also show that the vortex reconnection phase of the evolution is accelerated for moderate to strong ambient stratification $(1 \leq F r \leq 4)$. The same advection by the baroclinic vorticity that accelerates the Crow instability (for a smaller range of $F r$ ) later increases the vorticity gradients at the centerplane in and near plane $c$, enhancing the cross-diffusion that results in reconnection. For our $F r=2$ and $F r=1$ cases, the continuous advection by the baroclinic vorticity leads to the complete elimination of the threads, while for the larger $F r$ cases, threads continue to survive through very late times (until $t \simeq 11$ in the $F r=4$ case, and past the time our $\mathrm{Fr}=8$ and $\mathrm{Fr} \rightarrow \infty$ simulations were discontinued).

We have benefited from discussions with Philippe Spalart of Boeing Aircraft, Bob Robins of NorthWest Research Associates, Fazle Hussain of the University of Houston, and Øeyvind Andreassen of the Norwegian Defense Research Establishment. This work was supported by the Airforce Office of Scientific Research under grant F49620-98-1-0029 and the National Science Foundation under grant ATM-9419151. Computational resources were provided by the Pittsburgh Supercomputing Center under grant ATM96004P, and by NPACI at the San Diego Supercomputing Center and the 
University of Texas under grant MCA98N008.

\section{References}

1 Buntine, J.D. \& Pullin, D.I.: Merger and cancellation of strained vortices. J. Fluid Mech. 205 (1989), 263.

2 Canuto, C., Hussaini, M. Y., Quarteroni, A., \& ZAng, T. A.: Spectral methods in fluid dynamics. Springer (1988).

3 CRow, S.C.: Stability theory for a pair of trailing vortices. AIAA Journal 8 (1970), 2172.

4 Crow, S.C.: Motion of a vortex pair in a stably-stratified fluid. Poseidon Research Rep No. 1 (1970).

5 Garten, J.F.: An investigation of the effects of environmental stratification and shear on the evolution of vertically-propagating vortex pairs. PhD Thesis, University of Colorado (1997).

6 Garten, J.F., Arendt, S., Fritts, D.C., \& Werne, J.: Dynamics of counter-rotating vortex pairs in stratified and sheared environments. J. Fluid Mech. 361 (1998), 189.

7 Hussain, F.: Coherent structures and turbulence. J. Fluid Mech. 173 (1986), 303.

8 JADERBERG, L.K.: A method for computing the internal wave field around a nonlinear stratified flow. PhD Thesis, UCLA (1980).

9 Kida, R.M. \& Hussain, F.: Simulation of vortex reconnection. Physica D 37 (1989), 474.

10 Kida, S., TAkaoka, M., \& Hussain, F.: Collision of two vortex rings. J. Fluid Mech. 230 (1991a), 583.

11 Kida, S., TAKaOKA, M., \& Hussain, F.: Formation of head-tail structure in a two-dimensional uniform straining flow. Phys. Fluids A 3 (1991b), 2688.

12 Melander, M.V.\& Hussain, F.: Cut and connect of two antiparallel vortex tubes. CTR Report No. CTR-588 (1988), 257.

13 Melander, M.V. \& Hussain, F.: Cross-linking of two antiparallel vortex tubes. Phys. Fluids A 1 (1989), 633.

14 Moore, D.W.: Finite amplitude waves on aircraft trailing vortices. Aero. Q. 19 (1972), 307.

15 Olsen, J.H., Goldburg, A., \& Rogers, M.: Aircraft wake turbulence and its detection: Proceedings of a symposium on aircraft wake turbulence. Seattle (1971).

16 Pumir, A. \& Kerr, R.M.: Numerical simulation of interacting vortex tubes. Phys. Rev. Letters 58 (1987), 1636.

17 Robins, R.E. AND Delisi, D.P.: Numerical simulations of three-dimensional trailing vortex evolution. AIAA Journal 35 (1997), 1552.

18 Rogers , M.M. \& Moin, P.: The structure of the vorticity field in homogeneous turbulent flows. J. Fluid Mech. 176 (1987), 33.

19 Saffman, P.G.: A model of vortex reconnection. J. Fluid Mech. 212 (1990), 395.

20 SARPKAyA , Turgot: Trailing vortices in homogeneous and density-stratified media. J. Fluid Mech. 136 (1983), 85.

21 Scorer , R.S. \& Davenport, L.J.: Contrails and aircraft downwash. J. Fluid Mech. 43 (1970), 451.

22 Shelley, M.J., Meiron, D.I., \& Orszag, S.A.: Dynamical aspects of vortex reconnection of perturbed anti-parallel vortex tubes. J. Fluid Mech. 246 (1993), 613.

23 Spalart, P.R., Moser, R.D., \& Rogers, M.M.: Spectral methods for the Navier-Stokes equations with one infinite and two periodic directions. J. Comput. Phys. 96 (1991), 297.

24 Spalart, P.R.: On the motion of laminar wing wakes in a stratified fluid. J. Fluid Mech. 327 (1996), 139.

25 Spalart, P.R.: Airplane trailing vortices. Annu. Rev. Fluid Mech. 30 (1998), 107.

26 Tomassian, J.D.: The motion of a vortex pair in a stratified medium. PhD Thesis, UCLA (1979).

27 Virk, D., Hussain, F., \& Kerr, R.M.: Compressible vortex reconnection. J. Fluid Mech. 304 (1995), 47. 